\title{
Factores asociados con la gravedad de lesiones ocurridas en la vía pública en Cuernavaca, Morelos, México
}

Elisa del $\mathrm{C}$ armen $\mathrm{H}$ idalgo-Solórzano, $\mathrm{M}$ en $\mathrm{C}$, (1) Martha Hijar, PhD, ${ }^{(1)}$ Julia Blanco-Muñoz, M en $C_{\text {, }}^{(2)}$ María de la Luz Kageyama-Escobar, M en C. ${ }^{(1)}$

\section{Hidalgo-Solórzano EC, Híjar M, Blanco-Muñoz J, Kageyama-Escobar ML. \\ Factores asociados con la gravedad de lesiones ocurridas en la vía pública en Cuernavaca, Morelos, Mexico. Salud Publica Mex 2005;47:30-38. \\ El texto completo en inglés de este artículo está disponible en: http://www.insp. mx/salud/47/eng}

\section{Resumen}

Objetivo. Identificar los factores asociados con la gravedad de las lesiones ocurridas en la vía pública en personas que demandaron atención médica de urgencia en tres hospitales de la ciudad de Cuernavaca, Morelos, México. Material y métodos. Estudio transversal efectuado en la ciudad de Cuernavaca, estado de Morelos, durante los meses de febrero a abril de 2001. Incluyó individuos lesionados que demandaron atención a hospitales o que fallecieron en el lugar del accidente.Variables: edad, sexo, ingesta de alcohol, día y hora de ocurrencia, atención pre-hospitalaria, causa externa, tipo de lesión y gravedad con base en la Escala A breviada de Lesiones. Se utilizó análisis simple bivariado y multivariado. Resultados Se incluyeron 492 lesionados, de los cuales 23 fallecieron en el lugar. La principal causa externa fue accidentes de tránsito (52\%), $72 \%$ de los lesionados fueron leves. La variable aso ciada con gravedad fue accidentes de tránsito (RM 6.59, IC 95\% 2.52-17.23), ajustada por edad y escolaridad. Conclusiones Los accidentes de tránsito de vehículos de motor son los que provocan el mayor número de lesionados graves. El uso de la EscalaA Abreviada de Lesiones facilita el estudio de su magnitud y distribución de gravedad. El texto completo en inglés de este artículo está disponible en: http://www.insp.mx/salud/47/eng

Palabras clave: accidentes de tránsito; servicio de urgencia en hospital, México
Hidalgo-Solórzano EC, Híjar M, Blanco-Muñoz J, Kageyama-Escobar ML.

Severity of injuries in public streets

of an urban area in Mexico.

Salud Publica Mex 2005;47:30-38.

The English version of this paper

is available at: http://www.insp. $\mathrm{mx} / \mathrm{salud} / 47 / \mathrm{eng}$

\begin{abstract}
A bstract
Objective. To identify risk factors associated with severity of injuries occurring in public streets of an urban area. Material and Methods. Between February and A pril 2001, a cross-sectional survey was conducted among injured people seeking emergency care at three hospitals in C uernavaca, Morelos, Mexico. Information was also obtained for those who died on accident sites. Data on the following variables were collected: age, sex, alcohol intake, day and time of injury, prehospital care, external cause and nature of injury. The Abbreviated Injury Scale (AIS) was used to define severity of injuries. Data analysis consisted of descriptive, bivariate, and multivariate regression models. $\mathbf{R e}-$ sults. A total of 492 injured people were included in the study; 23 of them died on site. The main cause of injuries was road traffic accidents (52\%); $72 \%$ of injuries were not severe.Variables associated with the severity of injuries were: Road traffic accidents (odds ratio [OR] 6.59,95\% confidence interval $[\mathrm{Cl}] 2.52,17.23)$, adjusted for age and schooling. Conclusion. Road traffic injuries are the main cause of severe injuries. TheAIS is useful to assess the frequency and distribution of injury severity. The English version of this paper is available at: http://www.insp.mx/salud/47/eng
\end{abstract}

Key words: accidents, traffic; emergency service, hospital; Mexico

Este trabajo se realizó gracias al apoyo del Consejo $\mathrm{N}$ acional de Ciencia y Tecnología al proyecto 34472-M.

(1) Centro de Investigación en Sistemas de Salud, Instituto N acional de Salud Pública (IN SP) Cuernavaca, Morelos, México.

(2) Centro de Investigación en Salud Poblacional, IN SP Cuernavaca, Morelos, México.

Fecha de recibido: 20 de febrero de 2004 • Fecha de aprobado: 25 de agosto de 2004 Solicitud de sobretiros: D ra. Martha Híjar. Centro de Investigaciones en Sistemas de Salud, Instituto N acional de Salud Pública. Avenida Universidad 655, colonia Santa María A huacatitlan 62508 Cuernavaca, Morelos, México. Correo electrónico: mhijar@ correo.insp.mx 
as lesiones ocurren en todas las regiones y países del mundo y afectan a población de todos los grupos de edades. ${ }^{1}$ Constituyen un problema sanitario de creciente importancia en países de bajos ingresos, ${ }^{2}$ en los cuales el incremento en la industrialización y motorización trae como consecuencia modificaciones sociales y ambientales. ${ }^{3}$ Lo anterior ha provocado cambios importantes en la calidad de vida de la población, transformando o deteriorando sus niveles de salud. ${ }^{4}$

En México, como en muchos países en desarrollo, la pobreza y los retos de la transición epidemiológica hacen que las lesiones representen un grave problema de salud pública por el elevado costo que acarrean, tanto en vidas humanas ${ }^{5}$ como en atención médica. ${ }^{6}$

Las investigaciones realizadas en relación con el tema de las lesiones y la manera de prevenirlas ${ }^{7}$ han permitido identificar algunas asociaciones entre la ocurrencia de lesiones accidentales e intencionales con factores demográficos, económicos y sociales. ${ }^{8} \mathrm{Sin}$ embargo, en la mayoría de los casos, aquéllas se han enfocado en las consecuencias de las lesiones en términos de mortalidad, en alguna de sus causas ${ }^{9,10}$ o en un grupo de población específica.

En México, en lo que concierne a morbilidad y gravedad de las lesiones ${ }^{7,11,12}$ existen escasos estudios. Se sabe que la morbilidad por lesiones tiene un profundo efecto en los sistemas de salud, pues aumenta la demanda de atención en servicios de salud, sobre todo en las salas de urgencias. Además, la carga de los servicios no sólo se ejerce en la atención de las lesiones, sino que involucra a otras áreas como la rehabilitación, la atención psicológica, la recuperación de las capacidades productivas y la adaptación a las limitaciones orgánicas. ${ }^{13}$

Las características de la ciudad de Cuernavaca, que van desde su ubicación geográfica, así como el contar con una topografía de barrancas, determinan problemas como tener una estructura vial compleja, un crecimiento urbano de difícil control, la utilización inadecuada del suelo (invasión de aceras o banquetas por automóviles y puestos de venta), hasta la existencia de calles ensanchadas construidas a expensas de las aceras, generando en los peatones grandes distancias a cruzar, y creando inequidades en éstos, ya que son forzados a compartir el espacio con los vehículos. ${ }^{6}$ Por otra parte, la ciudad al ser un sitio de atracción turística ha generado el desarrollo de conductas y comportamientos de riesgo, que en otros contextos son más normados, por ejemplo: conducir bajo efectos de alcohol, y el manejar a altas velocidades, siendo los jóvenes la población que presenta los mayores daños a la salud provocados por lesiones debidas a accidentes de tráfico de motor.*

En relación con la gravedad de las lesiones se han estudiado dos grupos de clasificación basados en criterios anatómicos o fisiológicos. ${ }^{14}$ Estos comprenden índices y escalas que permiten cuantificar la gravedad en términos del compromiso que implica para la vida del lesionado, prever la discapacidad y la necesidad de rehabilitación.

Estas escalas o medidas de resumen son significativas en términos de decisión terapéutica y están relacionadas con el pronóstico del lesionado, la atención crítica y la reincorporación a sus actividades. Por lo anterior, la especificación de la gravedad de las lesiones constituye un elemento esencial para establecer medidas de prevención y control, una vez ocurrido el evento.

En este estudio, se utilizó la Escala Abreviada de las Lesiones (AIS, por sus siglas en inglés) por tratarse de un método numérico sencillo para medir y comparar la gravedad de las lesiones, en función de características propias del individuo, de su mecanismo de ocurrencia y de la demanda de atención médica y paramédica. ${ }^{15,16}$

El principal objetivo de este estudio es identificar los factores asociados con la gravedad de las lesiones ocurridas en la vía pública en personas que demandaron atención médica de urgencia en tres hospitales de la ciudad de Cuernavaca, Morelos, México, durante el año 2001.

\section{Material y métodos}

Se realizó un estudio transversal, en personas que fueron lesionadas en la vía pública de la ciudad de Cuernavaca, y que generaron demanda de atención médica en servicios de urgencia hospitalaria o que fallecieron como consecuencia de las mismas. El estudio se llevó a cabo durante los meses de febrero a abril de 2001, en tres hospitales de segundo nivel de atención, del estado de Morelos: Hospital Regional, del Instituto Mexicano de Seguro Social (IMSS), Hospital General José G. Parres, de la Secretaria de Salud (SSA) y Hospital Morelos (privado). En el caso de lesionados fallecidos en el lugar del evento, la información se obtuvo en el Servicio Médico Forense (SEMEFO).

* Inclán C, Híjar M, Tovar V. La identificación de los accidentes de tránsito como un problema local en Cuernavaca, México. El rol del capital social. Documento no publicado 2003. 
Los participantes que se seleccionaron durante el periodo del estudio corresponden al total de casos que demandaron atención médica de urgencias por haber sufrido una lesión en la vía pública o que fueron llevados al SEMEFO.

Debido a la dificultad de obtener información para determinar un tamaño de muestra que diera cuenta de la gravedad de las lesiones en la vía pública, se incluyeron todos los casos de lesionados como consecuencia de un evento accidental o intencional ocurridos en la vía pública, que demandaron atención médica de urgencias a las unidades mencionadas o que fueron llevados al SEMEFO, durante el periodo de estudio. El total de casos fue de 492 individuos, a quienes se les solicitó su consentimiento para participar en el estudio.

El protocolo de estudio fue evaluado y aprobado por la Comisión de Investigación, Ética y Bioseguridad del Instituto Nacional de Salud Pública.

La información fue obtenida mediante la aplicación de dos cuestionarios, (uno para lesionados y otro para fallecidos en el lugar del accidente) por encuestadores capacitados que permanecieron en los servicios de urgencias de los hospitales seleccionados, cubriendo las 24 horas del día, los siete días de la semana durante el período del estudio. La información obtenida del SEMEFO fue recopilada a través de personal médico y técnico.

Previamente se realizaron la estandarización de encuestadores y la prueba del instrumento durante una semana. Las preguntas incluidas en el instrumento han sido utilizadas por los autores en estudios previos sobre el tema de lesiones, en general, y de gravedad, en particular. ${ }^{6-8}$

Las unidades hospitalarias seleccionadas atienden pacientes lesionados en la vía pública, usuarios de servicios públicos o privados, así como asegurados. El periodo de estudio abarca la demanda de atención por lesiones durante periodos vacacionales (semana santa), fin de semana y días hábiles.

Se definieron las variables de la siguiente forma:

Variables sociodemográficas: edad, sexo, escolaridad, si tiene trabajo, si es derechohabiente.

Se incluyó la variable ingesta de alcohol la cual, tomando en consideración resultados de estudios previos, ${ }_{1}^{17}$ se midió por auto reporte, informando si hubo o no ingesta durante las seis horas previas al evento. No se midieron patrones de consumo de alcohol. Debido a razones médico legales y a situaciones locales del SEMEFO, no fue posible obtener información de esta variable en los casos que fallecieron en el lugar de ocurrencia del evento.
Variables relacionadas con la ocurrencia del evento (de la lesión): día de la semana, esta variable se dicotomizó en dos categorías: a) entre semana, que incluyó de lunes a jueves, $y \mathrm{~b}$ ) fin de semana y días festivos, que incluyó jueves y viernes santos y todos los días de viernes a domingo. Hora en que se produjo el evento, categorizándose como matutino, vespertino y nocturno. Variables relacionadas con la atención: si hubo atención prehospitalaria, personal que brindó la atención prehospitalaria. Tiempo transcurrido desde que ocurrió la lesión hasta que recibe atención médica.

Variables relacionadas con la lesión: tipo de lesión y región anatómica afectada, codificadas de acuerdo a lo establecido en la Clasificación Estadística Internacional de Enfermedades y Problemas Relacionados con la Salud Décima Revisión (CIE-10) del capítulo "Traumatismos, envenenamientos y algunas otras consecuencias de causas externas" (S00-T98). Las categorías de lesiones fueron:

Fracturas (S02, 12, 22, 32, 42, 52, 62, 72, 82, 92, T02, 08, $10,12,14.2)$

Luxación y esguince (S03, 13, 23, 33, 43, 53, 63, 73, 83, 93, T03, 09.2, 11.2, 13.2, 14.3)

Trauma cráneo-encefálico (TCE) (S00, 04-10, 14-20, 24$30,34-40,44-50,54-60,64-70,74-80,84)$

Otros traumas y contusiones (S00, 04, 09, 10, 14-16, 19, $20,24,25,29,30,34,35,39,40,44,45,46,49,50,54$, $55,56,59,60,64-66,69,70,74-76,79,80$, S84-89, 90, 94-96, 99, T00, 06, 07, 09.0, 09.3-09.5, 09.8, 09.9, 11.0, 11.3-T11.5, 11.8, 11.9, 13.0, 13.3-13.5, 13.8, 13.9, 14.0, 14.4-14.6, 14.8, 14.9)

Heridas: (S01, 11, 21, 31, 41, 51, 61, 71, 81, 91, T01, 09.1, $11.1,13.1,14.1)$

Causa externa: se codificó de acuerdo con lo establecido en la CIE-10 "Causas externas de morbilidad y de mortalidad" (V01-Y98) como:

Accidente de tránsito de vehículo de motor (incluye lesiones a peatones y ocupantes de vehículos de motor) (V01-09.2, 09.3, 12-V14 (cuarto carácter .3-.9), 19.4-19.9, 20-28 (cuarto carácter .3-.9), 29.4-29.9, 30-39 (cuarto carácter .4-.9), 40-49 (cuarto carácter .4- .9), 50-59 (cuarto carácter .4-.9), 60-69 (cuarto carácter .4-.9), 70-79 (cuarto carácter .4-.9), 80.3-80.5, $81.1,82.1,83.0-83.4,84.0-86,86.4,87.0-87.8,89.2,89.9)$

Otros accidentes como caídas en un mismo nivel y caídas de un nivel a otro, (W00-W19) accidentes causados por animales, (W53-W59) exposición al humo, fuego y llamas, (X00-X09) contacto con calor y sustancias calientes, (X10-X19).

Lesiones intencionales como: lesiones auto infligidas intencionalmente (X60-X84), agresiones (incluye ho- 
micidios, intento de homicidio, riñas, agresión sexual) (X85-Y09).

La variable dependiente fue gravedad de las lesiones, la cual se definió a partir de la AIS. Esta consiste en un índice anatómico que valora la gravedad mediante la descripción de las lesiones sufridas usando nueve regiones anatómicas (cabeza, cara, cuello, tórax, abdomen, médula espinal, extremidades superiores, extremidades inferiores y piel). ${ }^{15,16}$

La AIS establece puntuaciones de gravedad en una escala ordinal que van desde: 1 : leve, 2: moderada, 3 : serio, 4: severo, 5: crítico, hasta 6: muy grave (mortal.)

Para el análisis se categorizaron de la siguiente manera:

Lesión no grave: cuando las lesiones obtuvieron puntuaciones de 1 y 2 (contusiones, heridas y traumatismos superficiales, esguinces, luxaciones y fracturas simples).

Lesión grave: cuando las lesiones encontradas tuvieron puntuaciones que van desde 3 hasta 6 (fracturas expuestas de diferentes sitios, traumatismo cráneo encefálico, heridas penetrantes y trauma de tórax y abdomen).

\section{A nálisis de los datos}

En el análisis simple se calcularon proporciones para todas las variables, así como media y desviación estándar para la variable edad.

En el análisis bivariado se exploró la asociación entre cada variable independiente con la variable dependiente. Para la comparación se utilizó la prueba de Ji cuadrada, razón de momios cruda e intervalos de confianza de $95 \%$.

Finalmente, se generó un modelo multivariado utilizando regresión logística múltiple, incluyendo paso a paso en el modelo todas aquellas variables que en el análisis bivariado se asociaron con la gravedad de la lesión y cuya $p$ fue menor o igual a 0.20 . Se seleccionó el modelo más parsimonioso y que mejor explicaba el fenómeno en estudio.

Para el procesamiento de la información se utilizó el paquete estadístico Stata\%, versión 7.0.

\section{Resultados}

Del total de 492 casos de lesionados en la vía pública durante el periodo en estudio, $23(4.7 \%)$ fallecieron en el sitio donde ocurrió el evento y $32(6.5 \%)$ en la unidad hospitalaria.
La distribución de la demanda por institución fue: 239 (50.9\%) IMSS, 153 (32.6\%) SSA y 77 (16.4\%) Hospital Morelos. En el cuadro I se presentan las variables de acuerdo con el tipo de las mismas y con base en la causa externa, destacando que la principal causa de lesiones fueron los accidentes de tránsito, 251 (51.7\%). En relación con el sexo se encontró que por cada mujer lesionada que demandó atención médica de urgencias hubo 2.5 lesionados del sexo masculino. La media de edad para todos los lesionados fue de 30 años (DE 17 años). El grupo de edad más afectado fue el de 15 a 29 años. El 54.31\% de los lesionados contaban con educación media (secundaria, preparatoria o técnica), $57 \%$ de los lesionados tenían trabajo y $60 \%$ eran derechohabientes.

Respecto a la variable ingesta de alcohol se observa que para los casos que se reportaron como provocados por lesiones intencionales fue el grupo donde se encontró el porcentaje más alto, en relación con el total (98 personas) de aquellos que informaron haber ingerido bebidas alcohólicas en las seis horas previas a la ocurrencia del evento.

Durante los fines de semana y días festivos se presentó la mayor proporción de lesionados que demandaron atención médica $(56.5 \%)$; en el turno vespertino fue donde se observó el mayor porcentaje de casos.

Más de $50 \%$ de los lesionados recibieron atención prehospitalaria, proporcionada en su mayoría $(90.5 \%)$ por médicos y paramédicos. Llama la atención el alto porcentaje de casos donde la primera atención médica fue recibida en un lapso mayor a una hora después de ocurrido el evento.

Los principales tipos de lesión fueron otros traumas y contusiones $(32.4 \%)$. En relación con la gravedad de las lesiones, $28 \%$ de lesionados se clasificaron como graves, siendo mayor esta proporción en aquellos casos que resultaron de un accidentes de tránsito.

Para los lesionados que requirieron hospitalización $(21.6 \%)$, el promedio de estancia hospitalaria fue de $3.4 \pm 4.5$ días), y casi la mitad (49\%) presentó lesiones graves. En estos casos, el principal motivo de egreso fue la mejoría $(80 \%$.)

En el cuadro II se presentan las variables que se relacionaron con la gravedad de las lesiones ocurridas en la vía pública: edad de 60 y más, $R M$ cruda 2.10, IC95\% 0.99-4.30, escolaridad universitaria, $R M$ cruda 2.78, IC95\% 1.34-5.74, y la causa accidentes de tránsito, $R M$ cruda 2.46 , IC95\% 1.47-4.10.

En el cuadro III se muestra el modelo multivariado, donde se observa que el riesgo de lesiones graves se asocia con la causa accidentes de tránsito, $R M$ ajustada 6.59, IC95\% 2.52-17.23, ajustando por edad y 


\section{Cuadro I}

\section{Distribución de VARIABLES, SEgún CAUSA EXTERNA DE LESIONES EN LA Vía PÚBLICA.} Cuernavaca, Morelos, México, 2001

\begin{tabular}{|c|c|c|c|c|c|c|c|c|}
\hline \multirow{3}{*}{ Variables } & \multicolumn{6}{|c|}{ Causa externa } & \multirow{2}{*}{\multicolumn{2}{|c|}{ Total }} \\
\hline & \multicolumn{2}{|c|}{ Accidentes de tránsito } & \multicolumn{2}{|c|}{ Otros accidentes } & \multicolumn{2}{|c|}{ Lesiones intencionales } & & \\
\hline & $n$ & $\%$ & $n$ & $\%$ & $n$ & $\%$ & $\mathrm{n}$ & $\%$ \\
\hline \multicolumn{9}{|l|}{ Edad (años) } \\
\hline $1-14$ & 38 & 15.32 & 35 & 26.72 & 3 & 3.00 & 76 & 15.87 \\
\hline $15-29$ & 109 & 43.95 & 45 & 34.35 & 59 & 59.00 & 213 & 44.37 \\
\hline $30-59$ & 79 & 31.85 & 39 & 29.77 & 34 & 34.00 & 152 & 31.73 \\
\hline 60 y más & 22 & 8.87 & 12 & 9.16 & 4 & 4.00 & 38 & 7.93 \\
\hline \multicolumn{9}{|l|}{ Sexo } \\
\hline Masculino & 177 & 70.52 & 89 & 66.92 & 82 & 81.19 & 348 & 71.75 \\
\hline Femenino & 74 & 29.48 & 44 & 33.08 & 19 & 18.81 & 137 & 28.25 \\
\hline \multicolumn{9}{|l|}{ Escolaridad } \\
\hline $\mathrm{N}$ inguna-primaria & 64 & 32.32 & 40 & 36.70 & 21 & 27.63 & 125 & 32.64 \\
\hline Media & 98 & 49.49 & 61 & 55.96 & 49 & 64.47 & 208 & 54.31 \\
\hline Universitaria & 36 & 18.18 & 8 & 7.34 & 6 & 7.89 & 50 & 13.05 \\
\hline \multicolumn{9}{|l|}{ Trabaja } \\
\hline Sí & 125 & 58.96 & 50 & 45.45 & 55 & 67.90 & 230 & 57.00 \\
\hline No & 87 & 41.04 & 60 & 54.55 & 26 & 32.10 & 173 & 42.93 \\
\hline \multicolumn{9}{|l|}{ Derechohabiente } \\
\hline Sí & 113 & 57.07 & 72 & 66.06 & 50 & 60.98 & 235 & 60.00 \\
\hline No & 85 & 42.93 & 37 & 33.94 & 32 & 39.02 & 154 & 39.59 \\
\hline \multicolumn{9}{|l|}{ Ingesta alcohol } \\
\hline Sí & 32 & 15.17 & 21 & 16.67 & 42 & 42.86 & 95 & 78.16 \\
\hline No & 179 & 84.83 & 105 & 83.33 & 56 & 57.14 & 340 & 21.84 \\
\hline \multicolumn{9}{|l|}{ Día ocurrencia } \\
\hline Entre semana & 123 & 50.83 & 51 & 38.64 & 34 & 34.00 & 208 & 43.88 \\
\hline Fin de semana y festivo & 119 & 49.17 & 81 & 61.36 & 66 & 66.00 & 266 & 56.12 \\
\hline \multicolumn{9}{|l|}{ Hora ocurrencia } \\
\hline Matutino & 92 & 43.19 & 42 & 33.60 & 18 & 19.15 & 152 & 35.19 \\
\hline Vespertino & 92 & 43.19 & 69 & 55.20 & 43 & 45.74 & 204 & 47.22 \\
\hline Nocturno & 29 & 13.62 & 14 & 11.20 & 33 & 35.11 & 76 & 17.59 \\
\hline \multicolumn{9}{|l|}{ Atención prehospitalaria } \\
\hline Sí & 163 & 65.20 & 51 & 38.93 & 36 & 36.00 & 250 & 51.98 \\
\hline No & 87 & 34.80 & 80 & 61.07 & 64 & 64.00 & 231 & 48.02 \\
\hline \multicolumn{9}{|l|}{ Tiempo evento-atención } \\
\hline$<1$ hora & 68 & 35.23 & 28 & 27.18 & 22 & 27.16 & 118 & 31.30 \\
\hline$\geq 1$ hora & 125 & 64.77 & 75 & 72.82 & 59 & 72.84 & 259 & 68.7 \\
\hline \multicolumn{9}{|l|}{ Tipo de lesión } \\
\hline Fracturas cráneo/TCE & 54 & 21.51 & 12 & 9.09 & 14 & 13.86 & 80 & 16.53 \\
\hline Fracturas extremidades & 27 & 10.76 & 27 & 20.45 & 3 & 2.97 & 57 & 11.78 \\
\hline Luxaciones y esguinces & 33 & 13.15 & 17 & 12.88 & 2 & 1.98 & 52 & 10.74 \\
\hline Heridas & 48 & 19.12 & 39 & 29.55 & 51 & 50.50 & 138 & 28.51 \\
\hline Traumas y contusiones & 89 & 35.46 & 37 & 28.03 & 31 & 30.69 & 157 & 32.44 \\
\hline \multicolumn{9}{|l|}{ Gravedad } \\
\hline No grave & 160 & 64.26 & 106 & 81.54 & 77 & 77.78 & 343 & 71.76 \\
\hline Grave & 89 & 35.74 & 24 & 18.46 & 22 & 22.22 & 135 & 28.24 \\
\hline
\end{tabular}

TCE: trauma craneoencefálico 


\section{Cuadro II

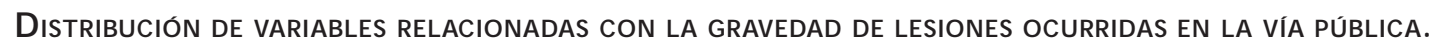 Cuernavaca, Morelos, México, 2001}

\begin{tabular}{|c|c|c|c|c|c|}
\hline \multirow{3}{*}{ Variables } & \multicolumn{4}{|c|}{ Gravedad de las lesiones } & \multirow{3}{*}{ RM Cruda (IC 95\%) } \\
\hline & \multicolumn{2}{|c|}{ No grave } & \multicolumn{2}{|c|}{ Grave } & \\
\hline & $n$ & $\%$ & $\bar{n}$ & $\%$ & \\
\hline \multicolumn{6}{|l|}{ Edad (años) } \\
\hline $1-14$ & 60 & 17.34 & 15 & 11.45 & $0.64(0.33-1.25)$ \\
\hline $15-29$ & 155 & 44.80 & 57 & 44.27 & $0.94(0.60-1.50)$ \\
\hline $30-59$ & 110 & 31.79 & 43 & 32.82 & 1 \\
\hline 60 y más & 21 & 6.07 & 17 & 11.45 & $2.10(0.99-4.30)$ \\
\hline \multicolumn{6}{|l|}{ Sexo } \\
\hline Masculino & 248 & 71.26 & 98 & 73.33 & $1.04(0.67-1.62)$ \\
\hline Femenino & 100 & 28.74 & 38 & 26.67 & 1 \\
\hline \multicolumn{6}{|l|}{ Escolaridad } \\
\hline $\mathrm{N}$ inguna-primaria & 105 & 32.71 & 18 & 30.00 & $1.14(0.6-2.17)$ \\
\hline Media & 180 & 56.07 & 27 & 45.00 & 1 \\
\hline Universitaria & 36 & 11.21 & 15 & 25.00 & $2.78(1.34-5.74)$ \\
\hline \multicolumn{6}{|l|}{ Trabaja } \\
\hline Sí & 194 & 57.91 & 37 & 56.06 & 1 \\
\hline No & 141 & 42.09 & 29 & 43.94 & $1.08(0.63-1.84)$ \\
\hline
\end{tabular}

Derechohabiente

\begin{tabular}{llllll} 
Sí & 206 & 63.00 & 32 & 53.33 & 1 \\
\hline No & 121 & 37.00 & 28 & 46.67 & $1.49(0.86-2.6)$
\end{tabular}

Ingesta alcohol

\begin{tabular}{lrrrrr} 
Sí & 76 & 23.03 & 19 & 19.42 & $0.75(0.42-1.3)$ \\
\hline No & 254 & 76.97 & 85 & 80.58 & 1
\end{tabular}

Día ocurrencia

\begin{tabular}{llllll} 
Entre semana & 146 & 42.57 & 62 & 47.69 & 1 \\
\hline Fin de semana / festivo & 197 & 57.43 & 68 & 52.31 & $0.81(0.54-1.21)$
\end{tabular}

Hora ocurrencia

\begin{tabular}{lrrrrr} 
Matutino & 108 & 34.18 & 43 & 38.05 & 1 \\
\hline Vespertino & 148 & 46.84 & 53 & 46.90 & $0.75(0.39-1.42)$ \\
\hline N octurno & 60 & 18.99 & 18 & 15.04 & $0.89(0.56-1.44)$
\end{tabular}

Atención prehospitalaria

\begin{tabular}{|c|c|c|c|c|c|}
\hline Sí & 190 & 54.60 & 60 & 45.80 & 1 \\
\hline No & 158 & 45.40 & 72 & 54.20 & $1.44(0.96-2.16)$ \\
\hline \multicolumn{6}{|l|}{ Tiempo evento-atención } \\
\hline$<1$ hora & 98 & 31.41 & 20 & 31.75 & 1 \\
\hline$\geq 1$ hora & 214 & 68.59 & 43 & 68.25 & $0.98(0.55-1.76)$ \\
\hline \multicolumn{6}{|l|}{ Causa externa } \\
\hline Accidentes de tránsito (CIE 10:V01-V89) & 160 & 46.65 & 89 & 65.93 & $2.46(1.47-4.10)$ \\
\hline 0 tros accidentes (CIE 10:W 00-W 19,W 53-W 59, X00-X 19) & 106 & 30.90 & 24 & 17.78 & 1 \\
\hline Lesiones intencionales (CIE 10: X60-Y09) & 77 & 22.45 & 22 & 16.30 & $1.26(0.66-2.4)$ \\
\hline
\end{tabular}

CIE 10: Clasificación Estadística Internacional de Enfermedades y Problemas Relacionados con la Salud. Décima Revisión. 


\section{Cuadro III Variables asociadas con la gravedad de lesiones ocurridas en la vía PÚBlica.* Cuernavaca, Morelos, México, 2001}

\begin{tabular}{lll} 
& \multicolumn{2}{c}{ Gravedad de las lesiones } \\
\cline { 2 - 3 } Variables & RM Ajustada & IC $95 \%$ \\
Causa externa & 6.59 & $2.52-17.23$ \\
Accidentes de tránsito & 1 & \\
\hline O tros accidentes & 1.11 & $0.28-4.38$ \\
\hline Lesiones intencionales & &
\end{tabular}

\begin{tabular}{cll} 
Edad (años) & 0.96 & $0.35-2.65$ \\
$1-14$ & 1.35 & $0.66-2.76$ \\
\hline $15-29$ & 1 & \\
\hline $30-59$ & 1.37 & $0.41-4.57$ \\
\hline 60 y más & &
\end{tabular}

\begin{tabular}{llc} 
Escolaridad & 1.18 & $0.52-2.67$ \\
N inguna-primaria & 1 & 1 \\
\hline Media & 2.22 & $1.01-4.88$ \\
\hline Universitaria & &
\end{tabular}

* N úmero de observaciones: 376, Log-likelihood:-145.32, LR $\chi^{2}$ (7):39.44, $p>\chi^{2}: 0.0000$, Pseudo R2: 0.1195

escolaridad, con un Log-likelihood de -145.32 y una $\mathrm{R}^{2}$ de 0.1195 .

\section{Discusión}

Las lesiones ocurren como fenómenos multicausales, que deben ser tratados como un problema de salud pública, no sólo por su influencia en el deterioro de las relaciones sociales ${ }^{18} \mathrm{y}$ productivas, sino porque producen daños a la salud. Es importante señalar que si bien el presente estudio parte de un diseño tipo encuesta transversal, su importancia radica en el hecho de que al haber incluido sólo aquellas lesiones que ocurren en la vía publica y, dentro de éstas, identificar que las provocadas por accidentes de tránsito son las más prevalentes en la población, la información que se obtiene sirve de base para el diseño de nuevas investigaciones que emplean metodologías que permitan definir sus determinantes.

Las unidades médicas públicas incluidas en este estudio (Hospital General José G. Parres, de la SSA y el Hospital Regional del IMSS) en la ciudad de Cuernavaca, además de ser unidades de segundo nivel de atención, son hospitales de concentración para toda la región. Asimismo, el Hospital Morelos es una institución privada a la que son canalizados los casos de lesio- nados en la vía pública, que cuentan con algún seguro de gastos médicos mayores. Este hecho permitió haber contado con un número de casos que nos aproxima a lo que está ocurriendo en Cuernavaca, en relación con la demanda de atención por lesiones a diversos tipos de unidades existentes en la ciudad.

Los resultados de este estudio indican que las lesiones ocurridas en la vía pública, sobre todo las debidas al tránsito de vehículos de motor, son las que provocan el mayor número de víctimas. Situación similar ha sido descrita por otros autores. ${ }^{2,6,19,20}$ Es importante destacar que la causa externa, específicamente los accidentes de tránsito, mostraron una asociación estadísticamente significativa con la gravedad de las lesiones. Tal asociación podría ser explicada principalmente por los factores que se relacionan con el tipo de fuerza involucrada. En el contexto de la vía pública se trata de un espacio donde el automóvil es hegemónico y desplaza a los demás usuarios, lo que favorece la creación de un espacio urbano deshumanizado, con tendencia a la marginación de peatones y a una desintegración social. ${ }^{21}$

Tanto los resultados obtenidos respecto a que la mayor proporción de lesionados que acudieron a los servicios de urgencias presentan lesiones no graves, así como que los jóvenes del sexo masculino es el grupo más afectado, concuerda con lo hallado por otros autores. ${ }^{6,22-24} \mathrm{Sin}$ embargo, respecto a la escolaridad, en este estudio la categoría licenciatura se encontró asociada con lesiones graves. Dicha asociación hasta ahora no ha sido publicada, lo que sugiere que esta variable puede estar correlacionada con la causa accidentes de tránsito, en especial con aquellos que acudieron a hospitales privados y que son cubiertos por aseguradoras. Este hecho deberá ser corroborado en estudios futuros.

En relación con el auto reporte de consumo de alcohol, en nuestro estudio se encontró una proporción similar a la comunicada por un hospital sobre pacientes que demandaron atención de urgencia por lesiones. ${ }^{25,26}$ Sin embargo, no fue posible demostrar la asociación entre consumo de alcohol y gravedad debido a que, en los casos que fallecieron en el sitio de ocurrencia, no se pudo medir esta variable, pues al momento de realizar el levantamiento de datos esta información no pudo ser recabada del SEMEFO aduciéndose motivos médico legales que impidieron su registro. Es importante tomar en cuenta este tipo de dificultades que pueden presentarse cuando se obtiene información de bases secundarias, para definir nuevas estrategias de recolección de información.

En lo que concierne a la atención prehospitalaria se ha reportado que una tercera parte de las muertes 
tardías secundarias a lesiones se deben a una inadecuada atención pre hospitalaria. ${ }^{19}$ En nuestro estudio el análisis de esta variable se limita a conocer su distribución por gravedad de lesiones y causa externa, sin que se puedan analizar las características de la misma en términos de calidad, oportunidad y experiencia de quien la proporciona. Lo anterior haría necesario un estudio dirigido específicamente a estos aspectos.

$\mathrm{Al}$ ser uno de los primeros estudios realizados en México donde se utiliza la Escala Abreviada de Lesiones para definir gravedad permitirá comparar sus resultados con investigaciones similares realizadas en otros contextos. ${ }^{16}$

Es importante señalar que si bien en nuestro estudio la causa accidentes de tránsito se encontró fuertemente relacionada con la gravedad de las lesiones en la vía pública, no se debe olvidar que esta causa engloba a conductores, peatones, ciclistas y motociclistas, todos ellos usuarios de la vía pública ${ }^{27}$ expuestos de manera diferenciada a sufrir lesiones o a morir por esta causa, lo que hace necesario realizar futuros estudios que permitan identificar los determinantes de gravedad en cada uno de estos actores.

Finalmente, tomando en cuenta los resultados de este estudio en el cual las lesiones que ocurren como consecuencia de un accidente de tránsito son las que más se asocian con gravedad y, además, se reconocen como la principal causa de muerte por lesiones en nuestro país, ${ }^{28}$ su prevención y control no pueden ni deben ser una competencia sólo del sector salud, sino una tarea de carácter intersectorial en la que todos los sectores involucrados se comprometan a ejercer acciones para evitar su ocurrencia.

\section{Agradecimientos}

Un agradecimiento especial a los Directores de los tres hospitales que participaron en el estudio: Hospital Regional, del IMSS, Hospital General José G. Parres, de la Secretaría de Salud y Hospital Morelos, de Cuernavaca.

\section{Referencias}

1. 0 rganización Panamericana de la Salud. Prevención de accidentes y lesiones. Serie PALTEX N 0.29.W ashington, DC: O PS; 1993.

2. Mock CN , A bantanga F, C ummings P, Koepsell TD. Incidence and outcome of injury in G hana: A commuity-based survey. Bull W orld Health 0 rgan, 1999;955-964.

3. Roberts lan. Reducing road traffic. BMJ 1998; 316: 242-243.
4. Langlo is JA, Keyl PM, Guralnik JM, Foley DJ, Marottoli RA, W allace RB. Characteristics of older pedestrians who have difficulty crossing the street.Am J Public Health 1997;87(3): 393-397.

5. Lozano R. El peso de la enfermedad en México: avances y desafíos. En: Frenk J, ed. 0 bservatorio de la salud: necesidades, servicios, políticas. México, DF: Fundación Mexicana para la Salud; 1997:23-61.

6. Híjar-Medina MC,Arredondo A, C arrillo C, Solórzano L. Road traffic injuries in an urban area in Mexico.An epidemiological and costs analysis. Accid A nal Prev 2004; 36: 37-42.

7. Híjar-Medina MC, Flores-Aldana ME, López-López MV. Cinturón de seguridad y gravedad de lesiones en accidentes de tráfico en carretera. Salud Publica Mex 1996; 38:118-127.

8. Híjar-Medina MC, Tapia-Yánez JR.A nálisis de la demanda por lesiones traumáticas a servicios de urgencia de 12 hospitales pediátricos de la Ciudad de México. Bol Med Hosp Infant Mex 1991; 48(10): 722-729. 9. López-López MV, Híjar-Medina MC, Rascón-Pacheco RA, BlancoMuñoz J. Muertes por homicidio, consecuencia fatal de la violencia. El caso de México, 1979-1992. Rev Saude Publica 1996; 30(1):46-52.

10. Híjar-Medina MC. U tilidad del análisis geográfico en el estudio de las muertes por atropellamiento. Salud Publica Mex 2000; 42: 188-193.

11. Híjar-Medina MC, Tapia-Yánez JR, López-López MV, Solórzano-Flores LI, Lozano-Ascencio R. Factores de riesgo de accidentes en niños. Estudio de casos y controles. Bol Med Hosp Infant Mex 1993;50(7): 463-474.

12. Híjar-Medina MC, Tapia-Yánes JR, López-López MV, Lozano-Ascencio R. El trabajo materno y la gravedad de lesiones accidentales en niños. Salud Publica Mex 1995; 37:197-204.

13. Cruz JM. La victimización por violencia urbana: niveles y factores asociados en A mérica Latina y España. Rev Panam Salud Publica 1999;5:259-267.

14. Prat-Fabregat S, González-Matheu E, Placer-López de Alda A, Siches C uadra C, Suso-Vergara S, Sánchez-Llo ret Tortosa J. Politraumatizados, ISS y control de calidad. Emergencias 1997; 9(2): 86-90.

15. Association for the Advancement of Automotive Medicine.The A bbreviated Injury Scale. 1990 Revision. D esplaines (IL), USA: Association for the advancement of automotive Medicine; 1998. 16. Stevenson M, Segui-G omez M, Lescohier I, Di Scala C, McD onaldSmith $G$.An overview of the injury severity score and the new injury severity score. Inj Prev 2001; 7:10-13.

17. Híjar-Medina M, C arrillo-O rdaz C, Flores-Aldana M,Anaya R, LópezLópez M. Factores de riesgo de lesión por accidentes de tráfico y el impacto de una intervención sobre la carretera. Rev Saude Publica 1999; 33 (5): 505-512.

18. Pellegrini-Filho A. La violencia y la salud pública. Rev Panam Salud Publica 1999; 5:219-21.

19. Trostchansky J. Los sistemas de trauma: un desafío asistencial. D isponible en: http://www.smu.org.uy/elsmu/institucion/comisiones/ Preacctra/sistemas\%20de\%20trauma.pdf. [2004 febrero 2].

20. Hussain LM, Redmond AD. Are pre-hospital deaths from accidental injury preventable? BMJ 1994; 308:1077-1080.

21. Tapia-Granados JA . La reducción del tráfico de automóviles: una política urgente de promoción de la salud. Rev Panam Salud Publica 1998; 3:137-151.

22. Hidalgo-Solórzano E.Aspectos epidemiológicos de la demanda de atención médica por lesiones causadas en accidentes de tráfico en México (tesis). México, DF: Instituto N acional de Salud Pública; 2002. 23. Correa MA, G onzález G, Herrera ME, O rozco A. Epidemio logía del trauma pediátrico en Medellín, Colombia 1992-1996. Colombia Med 2000; 31: 77-80.

24. Posada J, Ben-Michael E, Herman A, Kahan E, Richter E. D eath and injury from motor vehicle crashes in Colombia. Rev Panam Salud Publica 2000; 7:88-91. 
25. Borges G, Medina-Mora ME, Cherpitel Ch, Casanova L, Mondragón $L$, Romero M. Consumo de bebidas alcohólicas en pacientes de los servicios de urgencia de la ciudad de Pachuca, Hidalgo. Salud Publica Mex 1999; 41:3-11.

26. Rosovsky H.Accidentes y conducta violenta asociados a la ingesta de alcohol en la Ciudad de México: hacia una estrategia preventiva. Ponencia presentada en la Tercera Reunión Anual del Programa
Universitario de Medio Ambiente-UN AM;A cademia N acional de Medicina; 1994 octubre 19-21; México, D F, México.

27. Híjar-Medina MC, Trostle J, Bronfman M. Pedestrian injuries in Mexico:A multi-method approach. Soc Sci Med 2003; 57:2149-2159.

28. Centro $\mathrm{N}$ acional para la Prevención y Control de Accidentes. Compendio Estadístico de la Mortalidad Registrada por Accidentes, México, 1998-1999. México, DF: Secretaría de Salud; 1991. 